\title{
Características clínicas periodontales de piezas con diagnóstico de trauma de oclusión secundario
}

\author{
Periodontal clinical features of teeth with secondary trauma of occlusion \\ Yessica Janeth Torres-Cárdenas ${ }^{1, a}$, Beatriz del Carmen Chávez-Reátegui 1,a,b,c,;;,e;4, Jorge Enrique Manrique- \\ Chávez ${ }^{1, a, c ; 3, e ; 4}$
}

\section{RESUMEN}

Objetivos: Determinar las características periodontales de piezas con diagnóstico de trauma de oclusión secundario en pacientes atendidos en la Clínica Dental Docente de la Facultad de Estomatología "Roberto Beltrán" de la Universidad Peruana Cayetano Heredia durante los años 2011-2012. Material y métodos: Estudio retrospectivo, descriptivo, observacional y de corte transversal, con un universo conformado por pacientes con características clínicas de trauma de oclusión secundario recibidos por los estudiantes de pregrado. Resultados: Se incluyeron 110 historias clínicas de pacientes con diagnóstico de trauma de oclusión secundario, las cuales fueron evaluadas por según los criterios de inclusión y exclusión, quedando 95 casos como referencia para el presente estudio. Las piezas diagnosticadas con trauma de oclusión secundario representan mayor afectación en cuanto a la variable movilidad dental en el 98,09\% de los casos, pérdida del nivel de inserción clínica (NIC) moderada $(24,2 \%)$ y severa $(73,7 \%)$ y retención dental en el $100 \%$ de los casos. El tipo de pieza afectada por trauma de oclusión secundario es variable, con un 51,5\% de casos con afección sólo en piezas unirradiculares, 61,54\% sólo en piezas multirradiculares y $20,1 \%$ en ambos tipos de piezas. Presentándose con mayor prevalencia en los grupos etarios 41-50 (30,5\%), 51-60 (27,4\%) y 61-70 (24,2\%) años. Conclusiones: Las piezas diagnosticadas con Trauma de Oclusión Secundario representan un mayor compromiso en cuanto a la variable movilidad dental y retención dental, así como la pérdida de nivel de inserción clínica (NIC) severa, por lo que es de vital importancia valorar el estado periodontal de las piezas comprometidas con trauma de oclusión secundario para planificar una secuencia de tratamiento apropiada y determinar las posibilidades y limitaciones de la terapia periodontal.

PALABRAS CLAVE: Periodoncia, enfermedades periodontales, pérdida de la inserción periodontal, oclusión dental traumática.

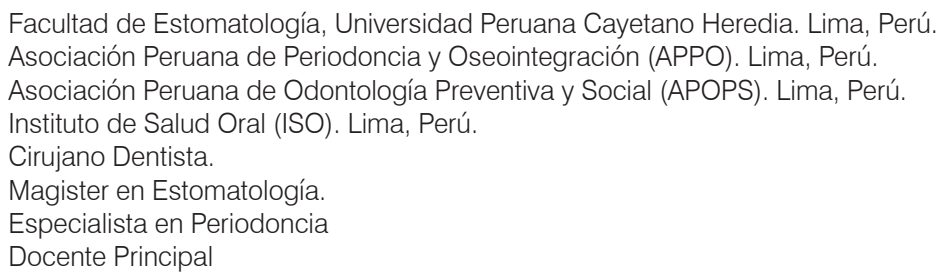




\section{SUMMARY}

Objectives: Determine the characteristics of periodontal diagnosis pieces Occlusion Secondary Trauma in patients treated at the Teaching Dental Clinic of the Faculty of Stomatology "Roberto Beltrán" UPCH during 2011-2012. Material and methods: It was retrospective, descriptive, observational and cross-sectional study, with a universe formed by patients with characteristics of Secondary Occlusal Trauma (Secondary Trauma from Occlusion) received by undergraduates. Results: Clinical history of 110 patients with a diagnosis of secondary occlusal trauma, which went through inclusion and exclusion criteria, leaving 95 cases as a reference for the present study. The teeth diagnosed with secondary occlusal trauma represent more unaffected in tooth mobility variable in the $98.09 \%$ of cases, loss of clinical attachment level moderate $(24.2 \%)$ and severe $(73.7 \%)$ and dental retention $100 \%$ of cases. The type of teeth affected by secondary occlusal trauma is variable, with $51.5 \%$ of cases single-rooted, $61.54 \%$ multi-rooted and $20.1 \%$ in both types. Though the highest prevalence in the 41 50 age group (30.5\%), 51-60 (27.4\%) and 61-70 (24.2\%). Conclusions: The teeth diagnosed with secondary occlusal trauma represent more affected evaluated as tooth mobility and tooth retention variable, and the loss of clinical attachment level severe, so it is important to assess the periodontal status of teeth engaged with secondary trauma occlusion to plan a sequence of appropriate treatment and determine the possibilities and limits of periodontal therapy.

\section{KEY WORDS: Periodontics, periodontal diseases, periodontal attachment loss, dental occlusion traumatic.}

\section{INTRODUCCIÓN}

La enfermedad periodontal es una enfermedad infecciosa-inflamatoria, que de acuerdo al grado de compromiso puede llevar a la pérdida total de los tejidos de soporte del diente. Siendo así particularmente alta en la población adulta, además de ser la principal causa de extracciones dentales en adultos mayores de 40 años (1).

El significado de trauma de la oclusión en el inicio de la periodontitis fue una fuente de controversia por décadas. Hoy sabemos de estudios experimentales que las fuerzas anormales de la oclusión no pueden provocar algún problema periodontal como la periodontitis (2-4).

La literatura clásica de los primeros años del siglo XX proveniente de autores como Karolyi (1901), Stillman (1917), Box (1935), Loe (1966) y Glickman (1962), citada por Albertini et al., consideraba como factor etiológico principal de la enfermedad periodontal al trauma oclusal, por tanto, cualquier alteración a nivel oclusal produciría periodontitis, de ahí el enfoque de realizar ajustes oclusales sobre las piezas comprometidas (5). Sin embargo, actualmente no se considera al diagnóstico de enfermedad periodontal como cofactor en su progresión ya que no provocaba por sí mismo la enfermedad, aunque sí es capaz de modificar su evolución o expresión (2-7).

Ya que el trauma de oclusión es la situación en la cual se producen lesiones de las estructuras de sostén, a consecuencia del acto de poner en contacto los maxilares, resultando así una lesión en el aparato de inserción como resultado de una fuerza oclusal excesiva (3). Es importante resaltar que el trauma de oclusión primario ocurre en un periodonto sano, el cual recibe fuerzas oclusales excesivas. En este contexto, ocurre algo totalmente diferente cuando el periodonto no se encuentra en condiciones normales ya que las fuerzas que reciben no van a tener un soporte periodontal adecuado, originando un trauma de oclusión secundario $(4,7-11)$.

Por ello, es sumamente importante realizar un buen diagnóstico clínico, así como conocer e identificar las causas del trauma de oclusión secundario, para realizar una correcta evaluación periodontal en pacientes con tal diagnóstico. Asimismo, es recomendable conocer los índices periodontales o indicadores clínicos que nos permitan identificar el grado de estado periodontal que presenta el paciente con esta afección. Todo esto considerando que aunque la enfermedad periodontal ocurre principalmente debido al crecimiento bacteriano en la gingiva o la formación de bolsas periodontales, esto puede verse afectado indirectamente por muchos otros factores de riesgos como el sistema vascular, reacción inflamatoria seve- 
ra y respuesta a la actividad inmunológica. $(1,11-14)$

De esta manera, el profesional puede valorar el estado periodontal de las piezas comprometidas con trauma de oclusión secundaria, planear una secuencia de tratamiento apropiado y determinar las posibilidades y limitaciones que pueda la terapia periodontal. Por tal motivo, la relación entre salud periodontal y trauma de oclusión secundario debe ser analizada con detenimiento para evaluar el estado de las piezas comprometidas al realizar el periodontograma y de ahí poder concientizar y orientar al paciente en el abordaje de su situación patológica de manera integral.

El objetivo del presente trabajo fue determinar las características periodontales de piezas con diagnóstico de trauma de oclusión secundario en pacientes atendidos en la Clínica Dental Docente de la Facultad de Estomatología "Roberto Beltrán" de la Universidad Peruana Cayetano Heredia (UPCH) durante los años 2011-2012.

\section{MATERIAL Y MÉTODOS}

Diseño de tipo retrospectivo, observacional, descriptiva y de corte transversal que estudió las características clínicas de pacientes con diagnóstico de trauma de oclusión secundaria que acudieron a la Clínica Dental Docente de la Universidad Peruana Cayetano Heredia (UPCH) durante los años 2011-2012. El tamaño del universo estudiado estuvo conformado por 110 historias clínicas, cuyos datos se encontraban almacenados en una base de datos previamente elaborada. Al aplicar algunos criterios de inclusión y exclusión sobre los registros, se quedó con un total de 95 historias clínicas para la elaboración de esta investigación. Los criterios de inclusión fueron: historias clínicas de pacientes recepcionados por los estudiantes de pregrado de la Facultad de Estomatología de la UPCH, de pacientes entre 18 a 70 años de edad y con diagnóstico de trauma de oclusión secundario. Los criterios de exclusión fueron: historias clínicas de pacientes con tratamiento ortodóncicos, con tratamientos médicos (últimos 6 meses), con tratamientos quirúrgicos, con enfermedad sistémicas y con diagnóstico de periodontitis o en tratamiento periodontal.

Se empleó una matriz de datos que contiene las siguientes variables: genero, edad, piezas dentarias afectadas por trauma de oclusión secundario, tipo de pieza afectada por trauma de oclusión secundario (unirradicular o multirradicular), pérdida de nivel se inserción clínica (NIC), compromiso de furcación, movilidad dentaria y retención dental.

Para el análisis descriptivo univariado y bivariado se utilizó la prueba estadística de Chi Cuadrado de Pearson. Se tomó en cuenta un valor de $\mathrm{p}<0,05 \mathrm{y}$ el intervalo de confianza del 95\% como el nivel de significancia estadística. Además se hizo un análisis descriptivo de las variables en tablas de frecuencias y porcentajes.

El protocolo de investigación fue revisado y aprobado por las autoridades de la Facultad de Estomatología y Departamento Académico de Clínica Estomatológica (DACE) para acceder a la información de la base de datos de las historias clínicas y por el Comité Institucional de Ética (CIE) de la Universidad Peruana Cayetano Heredia (UPCH).

\section{RESULTADOS}

Se evaluaron 95 historias clínicas de pacientes con diagnóstico de trauma de oclusión secundario atendidos por los alumnos de pregrado de la Clínica Dental Docente de la UPCH. Con respecto a la variable sexo, se evidencio un $64,2 \%$ para el sexo femenino y un $35,8 \%$ para el sexo masculino. La edad media fue de 52 años, donde 30 años es la edad mínima y 73 años es la edad máxima. Los grupos etarios con mayor frecuencia fueron los de 40-44 (30,5\%) años y 55-59 años $(27,4 \%)$, siendo de menor frecuencia los mayores de 71 años con solo $1,1 \%$.

La distribución de pacientes según cantidad de piezas afectadas se encuentra distribuida en una media de 3,81 , donde 1 es el número mínimo de pieza afectada y 15 es el número máximo de piezas afectadas. En el cual existe mayor prevalencia entre $2-4$ piezas. Así mismo se observó un paciente que tuvo un dato extremo (outlier) y que presentó 15 piezas dentarias afectadas (Tabla 1).

La distribución según la variable tipo de pieza afectada con trauma de oclusión secundaria fue de 51,5\% para las piezas unirradiculares, seguido de $28,4 \%$ para las piezas multirradiculares con 27 piezas y finalmente con un $20,1 \%$ para ambos tipos de piezas con 19 casos de todo la población estudiada. 
Tabla 1. Características de los pacientes con trauma de oclusión secundario atendidos en la Clínica Dental Docente de la UPCH, años 2011-2012.

\begin{tabular}{cccc}
\hline VARIABLE & CATEGORÍA & $\mathbf{N}^{\circ}$ & $\mathbf{\%}$ \\
\hline \multirow{2}{*}{ Género } & Femenino & 61 & 64,2 \\
& Masculino & 34 & 35,8 \\
Grupo Etario & $30-40$ años & 16 & 16,8 \\
& $41-50$ años & 29 & 30,5 \\
& $51-60$ años & 26 & 27,4 \\
Higiene Oral & $61-70$ años & 23 & 24,2 \\
& Más de 71 años & 1 & 1,1 \\
& & & \\
& Adecuada & 14 & 10,77 \\
& Aceptable & 55 & 42,31 \\
& Deficiente & 61 & 46,92 \\
Tipo de pieza afectada & & & \\
& Unirradicular & 49 & 51,5 \\
& Multirradicular & 27 & 61,54 \\
& Ambas & 19 & 20,1 \\
\hline
\end{tabular}

Según la prueba Chi Cuadrado, se evidenció que las variables tipo de piezas afectadas con trauma de oclusión secundario y número de piezas afectadas con trauma de oclusión secundario no se encuentran relacionadas $(\mathrm{p}>0,05)$. Se encontró que la afectación en el grupo de 1-2 piezas dentarias con trauma de oclusión secundario se encuentra en mayor frecuencia en piezas de tipo multirradicular con 19 pacientes (70,3\%), en el grupo de 3-4 piezas y de 5-6 piezas con trauma de oclusión secundario se encontró con mayor afectación a las piezas de tipo unirradicular con 19 casos $(38,7 \%)$ y 11 casos $(22,4 \%)$ respectivamente, en el grupo de 7-8 piezas afectadas con trauma de oclusión secundario se encontró la mayor frecuencia en ambos tipos de piezas en 5 pacientes $(26,31 \%)$ y finalmente en el grupo de $>8$ piezas afectadas con trauma de oclusión secundario se encontró solo un paciente tanto en piezas de tipo unirradicular y multirradicular.

Se evidenció la variable retención dental en un $100 \%$ de los casos y aplicando la prueba Chi Cuadrado se es- tableció que las variables furcación y número de pieza afectada no se encontraban relacionadas $(p>0,05)$. De igual forma, se encontró presencia de furcación en piezas multirradiculares en solo 1 caso $(3,7 \%)$ de toda nuestra población de estudio. La distribución según la variable movilidad dentaria se encontró en un $98,9 \%$ de los casos (grado 1 en el $8,4 \%$, grado 2 en el $44,2 \%$ y grado 3 en el $46,3 \%$ de casos) en tanto que la ausencia de movilidad fue en el 1,1\% de casos.

Por otro lado, la variable según pérdida de nivel inserción clínica (NIC) se encuentra distribuida en un $73,7 \%$ para el estado severo, un $24,2 \%$ el estado moderado y en un $2,1 \%$ el estado leve. Se evidenció que las variables pérdida de NIC y sexo no se encuentran relacionadas $(\mathrm{p}>0,05)$, donde la pérdida de NIC leve se presenta más en el sexo femenino $(2,11 \%)$, pérdida de NIC moderado en pacientes de sexo masculino $(17,89 \%)$ y pérdida de NIC severo también en los de sexo masculino (46,32\%) (Tabla 2$)$.

Se evidenció según la prueba Chi Cuadrado que las 
Tabla 2. Variables periodontales de las piezas con trauma de oclusión secundario de pacientes atendidos en la Clínica Dental Docente de la UPCH, años 2011-2012.

\begin{tabular}{cccc}
\hline VARIABLE & CATEGORÍA & $\mathbf{N}^{\circ}$ & $\mathbf{\%}$ \\
\hline \multirow{2}{*}{ Retención dental } & Presenta & 95 & 100 \\
& No Presenta & 0 & 0 \\
Pérdida de NIC & Leve & 2 & 2,1 \\
& Moderada & 23 & 24,2 \\
& Severa & 70 & 73,7 \\
Movilidad & Presenta & 94 & 98,09 \\
& No Presenta & 1 & 1,1 \\
Furcación & & & 3,07 \\
& Presenta & 1 & 96,03 \\
& No Presenta & 26 & \\
\hline
\end{tabular}

variables NIC y movilidad dental no se encontraban relacionadas $(\mathrm{p}>0,05)$. Pacientes con pérdida de NIC leve presentaron movilidad dentaria en un $2,11 \%$, pacientes con NIC moderado presentaron movilidad en un $23,16 \%$ y pacientes con pérdida de NIC severa presentaron movilidad en un $73,68 \%$. De igual forma, las variables pérdida de NIC y rango etario tampoco se encuentran relacionadas $(p>0,05)$. Pacientes de 30 a 40 años presentaron pérdida de NIC severa en $9,47 \%$, pacientes entre 41 a 50 años presentaron pérdida de NIC severa en un $22,11 \%$, pacientes entre 51 a 60 años presentaron pérdida de NIC severa en un $22,11 \%$ y pacientes mayores de 71 años presentaron pérdida de NIC moderada en un $1,05 \%$.

Finalmente, se evidenció según la prueba Chi Cuadrado que las variables pérdida de NIC y número de pieza afectada con trauma de oclusión secundaria no se encuentran relacionadas $(\mathrm{p}>0,05)$. Pacientes con pérdida de NIC leve se presentan sólo en 2 piezas multirradiculares $(7,4 \%)$, pacientes con pérdida de NIC moderado se presenta en su mayoría en piezas unirradiculares $(24,4 \%)$ y las piezas con pérdida de NIC severo se presentan en su mayoría en piezas unirradiculares $(75,5 \%)$.

\section{DISCUSIÓN}

En nuestro estudio existe una prevalencia mayor del sexo femenino con trauma de oclusión secundario $(64,2 \%)$ en comparación al sexo masculino $(35,8 \%)$. Dentro del grupo etario, se encontró una mayor prevalencia en pacientes entre 41-50 años representado un $30,5 \%$ de los casos. Estos resultados coinciden con los de le Jin, quién asoció la severidad de la periodontitis con el trauma de oclusión con mayor frecuencia en pacientes de sexo femenino (periodontitis crónica avanzada en mujeres mayores de 30 años) (15).

Con respecto al número de piezas afectadas con trauma de oclusión secundario, nuestro estudio revela que la mayor afectación se encuentra en 2-4 piezas que representa a un $21,1 \%$ del total de casos. Además, el tipo de pieza afectada con mayor frecuencia fueron las piezas unirradiculares con $51,5 \%$, seguidos de las multirradiculares con $28,4 \%$. En lo que respecta al estado de afectación del NIC, encontramos con mayor afectación al grado severo en un 73,7\%, moderado en un $24,2 \%$ y leve en un $2,1 \%$ de los casos. Estos resultados están relacionados con lo propuesto por Gamonal, quién en dos examinaciones simultáneas en el tiempo, explicó que si al menos dos sitios 
presenten pérdida de inserción proximal de $\geq 3 \mathrm{~mm}$ y con signos de inflamación sangrado al sondaje y profundidad al sondaje, serán considerados que presentan actividad y pueden seguir perdiendo inserción; lo cual conllevaría a un mayor grado de afectación y posible afectación y pérdida de piezas dentales (16).

Con respecto a la movilidad dentaria, los resultados revelan que el 98,9\% de casos presentan esta característica, siendo la más prevalente la movilidad grado 2 y 3 en $44,2 \%$ y $46,3 \%$ respectivamente. Esto puede deberse a alguna fuerza masticatoria inadecuada o algún deterioro del tejido de soporte. Dado que el trauma de oclusión se genera producto de una fuerza masticatoria inadecuada, nuestros resultados coinciden con el de Nunn y Harrel quienes concluyeron que los dientes con discrepancia oclusal contaban con una profundidad al sondaje considerable, además de recesiones gingivales y una movilidad significativamente peor que los dientes sin discrepancia oclusal; esto respaldado por la literatura clásica de Halozonetis en su estudio sobre la relación de los parámetros clínicos de la pérdida de inserción en subgrupos de sujetos con enfermedad periodontal destructiva $(17,18)$.

Por otro lado Apocada menciona que cuando la intensidad de las fuerzas oclusales aumentan, el periodonto sufre engrosamiento de las fibras del ligamento periodontal y aumento de la densidad del hueso alveolar; así podemos relacionar, según nuestros resultados, la alta prevalencia de movilidad dentaria en los casos de estudio, erste aumento de movilidad dental puede tener un efecto negativo en las condiciones ecológicas en la bolsa, en especial debido a los patógenos periodontales pueden proliferar (6).

De igual forma, en relación a la movilidad dentaria, Philstrom menciona que Bruce evaluó la asociación entre signos de trauma de oclusión secundaria y severidad de la periodontitis y concluyó que los dientes con mayor movilidad dentaria y ensanchamiento del espacio para el ligamento periodontal tienen mayor profundidad al sondaje y mayor pérdida de NIC (19). Por otro lado, Branschofsky menciona que la prevalencia del trauma de oclusión secundaria esta positivamente correlacionada con la pérdida de inserción y la severidad de la periodontitis (20).

Según la retención de placa, el 100\% de casos presenta esta característica siendo la más frecuente del gru- po de estudio debido a su higiene oral desfavorable y/o la falta de conocimiento de una adecuada higiene oral, lo cual es respaldado por la literatura clásica de Loe (21). Los resultados del presente estudio se respaldan en lo propuesto por Stephen K. quién sostiene que la salud periodontal depende del equilibrio entre un medio controlado sistémicamente que gobierna el metabolismo tisular y el ambiente externo del diente; donde la oclusión es también un componente importante (22). Asimismo, Lindhe menciona que la formación de placa blanda y calcificada es favorecida por la ubicación de los conductos excretores de las glándulas salivales mayores, ya que el calcio y fosfato de la saliva promueven su mineralización; estos acúmulos actúan como reservorios de bacterias que a largo plazo generan deterioro periodontal que puede conllevar a la pérdida de soporte periodontal, por lo que se observa clínicamente grados de movilidad desfavorables con posibles pérdidas dentarias (3).

Según el estado de furcación, los resultados revelan que el 98,9\% del grupo de estudio no la presenta, eso puede deberse al menor número de piezas multirradiculares comprometidas. Sin embargo, el estudio de Cattabriga demuestra que las primeras molares superiores son más frecuentemente afectadas que las primeras molares inferiores (23). Ikeda refiere que un defecto de furcación se produce a partir de la destrucción de los tejidos de soporte de dientes multirradiculares, permitiendo el acceso clínico o incluso la visualización del área interradicular de manera parcial o total. La etiología principal de esta destrucción tisular es la enfermedad periodontal, pero existen otras causas y factores asociados a esta alteración (24).

De acuerdo a los resultados de este estudio podemos extraer una serie de indicadores de uso clínico habitual en la práctica diaria que nos ayudarán a plantear estrategias de abordaje y solución a los diversos factores causantes y agravantes del trauma de oclusión secundario, considerando que el trauma de oclusión es resultado de las fuerzas anormales de la oclusión que pueden llegar a producir lesiones de las estructuras de sostén a consecuencia del acto de poner en contacto los maxilares, debido al estado de salud periodontal del paciente.

Los resultados de la investigación corroboran la literatura existente, por lo que podemos concluir que es de vital importancia, por parte del clínico opera- 
dor, valorar el estado periodontal de las piezas comprometidas con trauma de oclusión secundario para planificar una secuencia de tratamiento apropiada y determinar las posibilidades y limitaciones que puedan tener en su terapia, para resolver el problema de salud periodontal mediante procedimientos clínicos en periodoncia previamente descritos.

\section{Correspondencia:}

Beatriz del Carmen Chávez-Reátegui

Correo electrónico:

beatriz.del.carmen.chavez@upch.pe

\section{REFERENCIAS BIBLIOGRÁFICAS}

1. Wolf H, Hassell T. Atlas a color de periodontología. Bogota: Amolca; 2009.

2. William W. Occlusal trauma: Effect and impact in the periodontium. Ann Periodont. 1999; 4:102-6.

3. Lindhe J, Karring T, Lang N. Periodontología clínica e implantología odontológica. 5ta Ed. Singapore: Blackwell Munksgaard; 2008.

4. Dale B. Periodontal epithelium: a newly recognized role in health and diseases. Periodontal. 2000. 2002;30:70-8.

5. Albertini G, Bechelli D, Capusotto A. Importancia del diagnóstico y tratamiento multidisciplinario del Trauma Periodontal por Oclusión. Rev Asoc Odontol Argent. 2007; 95(2):157-71.

6. Apodaca A. Fundamentos de la oclusión. Ciudad de México: Editorial Tresguerras; 2004.

7. Wilson T, Kormman K. Fundamentals of periodontics. Singapor: Ed Quintessences Book; 1992.

8. Bosshardt D. The junctional epithelium: From health disease. J Dent Res. 2005; 84:9-20.

9. Mueller H. Periodontología. Ciudad de México: Editorial El Manual Moderno; 2006.

10. Newman M,TakeiH,KlokkevoldP.Periodontologíaclínica de Carranza. 11va Edición. Caracas:Amolca; 2014.

11. Hallmon W. Occlusal trauma: Effect and impact on the periodontum. Ann Periodontol. 1999; 4:102-7.

12. Foz A. Occlusal adjustment associated with periodontal therapy: A systematic review. Journal of Dentistry. 2012; 40(12): 1025-35.

13. Pegoraro L, Rodríguez P. Prótesis fija: Patologías oclusales y disfunciones craneomandibulares. Sao Paulo: Editorial Artes Médicas; 2001.

14. Rateistschack K, Wolf H. Color Atlas of periodontology. New York: George Thieme Verlad; 1985.

15. Jin L. Clinical diagnosis of trauma from occlusion and its relation with severity of periodontitis. J of Clinic Periodontology. 2005; 19(2): 92-97.

16. Gamonal J, Bascones A, Jorge O, Silva A. Chemokine in gingival crevicular fluid of adult patients with periodontitis. J Clin Periodontol. 2000; 27:675-81.

17. Halozonetis T. Relationship of clinical parameters to attachment loss in subsets of subject with destructive periodontal disease. J Clin Periodontol. 1989; 16:543-68.

18. Nunn ME, Harrel SK. The effect of oclusal discrepancies on periodontics: Relationship of initial oclusal discrepancies to initial clinical parameters. J Periodontol. 2001;72(4): 485-94.

19. Philstrom L. Association between Signs of Trauma from Occlusion and Periodontitis. Journal of Periodontology. 1997;57 (1):1-6.

20. Branschofsky M. Secondary trauma from occlusion and periodontitis. Quintessence I. 2011; 42(6):512-22.

21. Loe H, Anerud A., Boysen H. The natural history of periodontal disease in man: prevalence, severity, extent of gingival recession. J Periodontol. 1992; 63:489-95.

22. Stephen K. Fuerzas oclusales como factor de riesgo de enfermedad periodontal. Art Periodontology. 2003; 4: 111-17.

23. Cattabriga M, Pedrazzoli V, Wilson TG Jr. The conservative approach in the treatment of furcation lesions. Periodontol. 2000; 22:133-53.

24. Ikeda A, Mori G. Defectos de furcación: Etiología, diagnóstico y tratamiento. Rev Estomatol Herediana. 2012; 20(3):172-8.

Recibido : 11/09/2015 Aceptado: 28/04/2016 